\title{
WORKFORCE Identifying positive change within the NHS as a result of the COVID-19 pandemic
}

\author{
Authors: Rachel Barr-Keenan, ${ }^{A}$ Tayla Fay, ${ }^{B}$ Aleksander Radulovic ${ }^{C}$ and Sanjana Shetty ${ }^{D}$
}

\section{Objective}

We aimed to identify positive change within the NHS as a result of the COVID-19 pandemic by assessing staff views on re-deployment, wellbeing and workplace satisfaction.

Design

An anonymous questionnaire was sent out to medical staff working across four major NHS trusts using SurveyMonkey.

\section{Setting}

We surveyed staff working in NHS trusts across London and the surrounding areas.

\section{Participants}

We used a randomly selected range of medical, nursing and dental staff across multiple specialties, including those who were redeployed and those who were not.

\section{Main outcome measure}

We reviewed positive and negative responses to binary questions.

Staff surveyed felt a greater sense of workplace camaraderie and fellowship, and generally more appreciated as NHS caregivers than they had prior to the COVID-19 pandemic. It is clear that the pandemic has been a catalyst for reflection, as a majority of staff $(71.43 \%)$ felt there was more mental health and wellbeing support accessible in their workplace now than there was a year ago, and $75.63 \%$ felt that COVID-19 had influenced their perspective on work-life balance.

\section{Conclusion}

The first wave of the pandemic engendered positive change, and positive staff attitudes persisted in the face of a second wave due to ascribed support, appreciation, and co-worker camaraderie and fellowship.

KEYWORDS: COVID-19 pandemic, mental health, attitudes of healthcare personnel, work-life balance, work performance

DOI: $10.7861 /$ fhj.2021-0079

\footnotetext{
Authors: ${ }^{\text {A }}$ clinical fellow in oral and maxillofacial surgery, The Royal London Hospital, London, UK; ${ }^{\mathrm{B}}$ newborn intensive care unit clinical fellow, University College London Hospitals NHS Foundation Trust, London, UK; ' medical student, University College London Hospitals NHS Foundation Trust, London, UK; ' ear, nose and throat senior house officer, Portsmouth Hospitals University NHS Trust, Portsmouth, UK
}

\section{Introduction}

At the time of writing, over 111,000 people in the UK have died of COVID-19 since 02 March 2020.' SARS-CoV-2 has been detected in more than 3.9 million people in the UK with, at one point, 4,077 people throughout UK hospitals simultaneously requiring mechanical ventilation. ${ }^{1,2}$ To respond to the increasing number of COVID-19 patients requiring intensive care support, the NHS redeployed staff between departments. Staff had to learn new skills and adapt to very new roles.

Low staff morale and burnout are long-reported problems in the $\mathrm{NHS}$ and have to be related to patient outcomes and the quality of care provided. ${ }^{3}$ The 2019 NHS staff survey demonstrated that $27.7 \%$ of NHS staff interviewed did not feel respected and that $77.1 \%$ occasionally, or frequently, experienced unrealistic time pressures. ${ }^{4}$ These long-established issues that plague the NHS, coupled with the known negative impacts of pandemics and the novelty of redeployment, personal protective equipment (PPE) shortages and lockdowns, have created an unprecedented environment that NHS staff have been asked to accept.

The pandemic has forced NHS teams to promptly address some of these long-standing issues and implement positive change. It is hypothesised within this study that, despite the concerns already mentioned, this pandemic has resulted in many positive and necessary changes within the NHS that will result in improved mental wellbeing and satisfaction of staff. The study endeavoured to identify staff perceptions, positive impacts and changes brought to the NHS within the first wave of the COVID-19 pandemic. It also sought to assess staff opinion prior to facing future waves.

It is known that previous health crises have negatively affected the psychological welfare of healthcare practitioners. Following the $2002-2004$ SARS epidemic, as many as $10 \%$ of employees from a Beijing hospital experienced symptoms of post-traumatic stress disorder. ${ }^{5}$ Similar negative outcomes on both the mental and physical health of healthcare professionals involved with COVID-19 patients have been published. ${ }^{6-10}$ The impact of these very challenging conditions was recognised early and, in response, trusts across the UK have introduced increased support for staff, including hotlines, relaxation hubs, resources on self-management, home-working support and mindfulness sessions. ${ }^{11-15}$

\section{Methods}

Survey

A survey was presented as a five-page, online SurveyMonkey questionnaire with 19 questions. It took an average of 2 minutes 
8 seconds to complete. The use of SurveyMonkey allowed this to be safely distributed to all staff, avoiding face-to-face contact, and ensuring complete anonymity. All questions were optional. The survey is shown in supplementary material S1. Invitations for the surveys were distributed between 17 November 2020 to 19 December 2020

\section{Participants}

One-hundred and thirty-one members of staff across four different hospital trusts (Barts Health NHS Trust, Guy's and St Thomas' NHS Foundation Trust, Portsmouth Hospitals University NHS Trust and University College London Hospitals NHS Foundation Trust) participated. We employed a multidisciplinary approach, obtaining responses from nursing, medical and dental staff across multiple specialties, regardless of whether they had been redeployed in the first wave or not.

\section{Ethical approval}

Approval was not sought or required for this study because the data collection was anonymous and undertaken by medical staff to evaluate the current services without randomisation. There was no patient involvement in this study.

\section{Results}

A total of 131 members of staff across the four named hospital trusts were surveyed. As all questions were optional, the data analysis for each was carried out independently, only logging the provided answers.

The first aim of the study was to establish staff perception of redeployment and so the proportion of redeployed and non-redeployed members of surveyed staff was established (Fig 1).

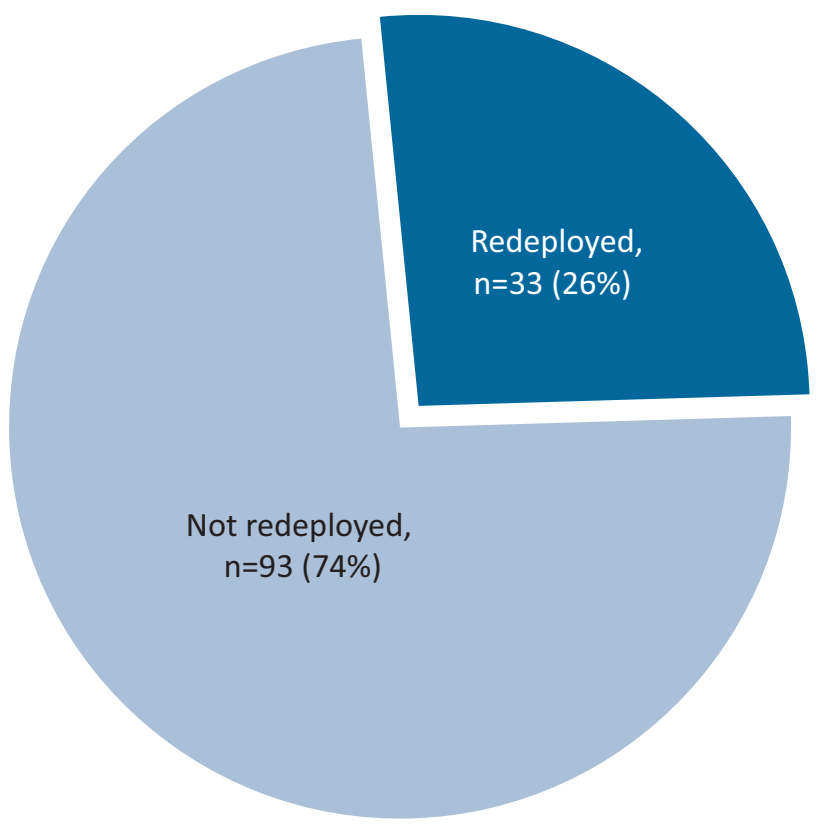

Fig 1. The proportion of the total cohort interviewed, $n=126$.

Out of the 126 participants, $26.19 \%$ were redeployed during the first wave of the COVID-19 pandemic and $73.81 \%$ remained in their usual posts.

The experiences of redeployment were then examined within this cohort of participants.

Our analysis demonstrated that $64.71 \%$ felt comfortable in their new role and $35.29 \%$ did not (Fig 2). Similarly, $66.67 \%$ felt valued during redeployment and $68.75 \%$ felt that they had gained positive experiences that they would be able to translate to their

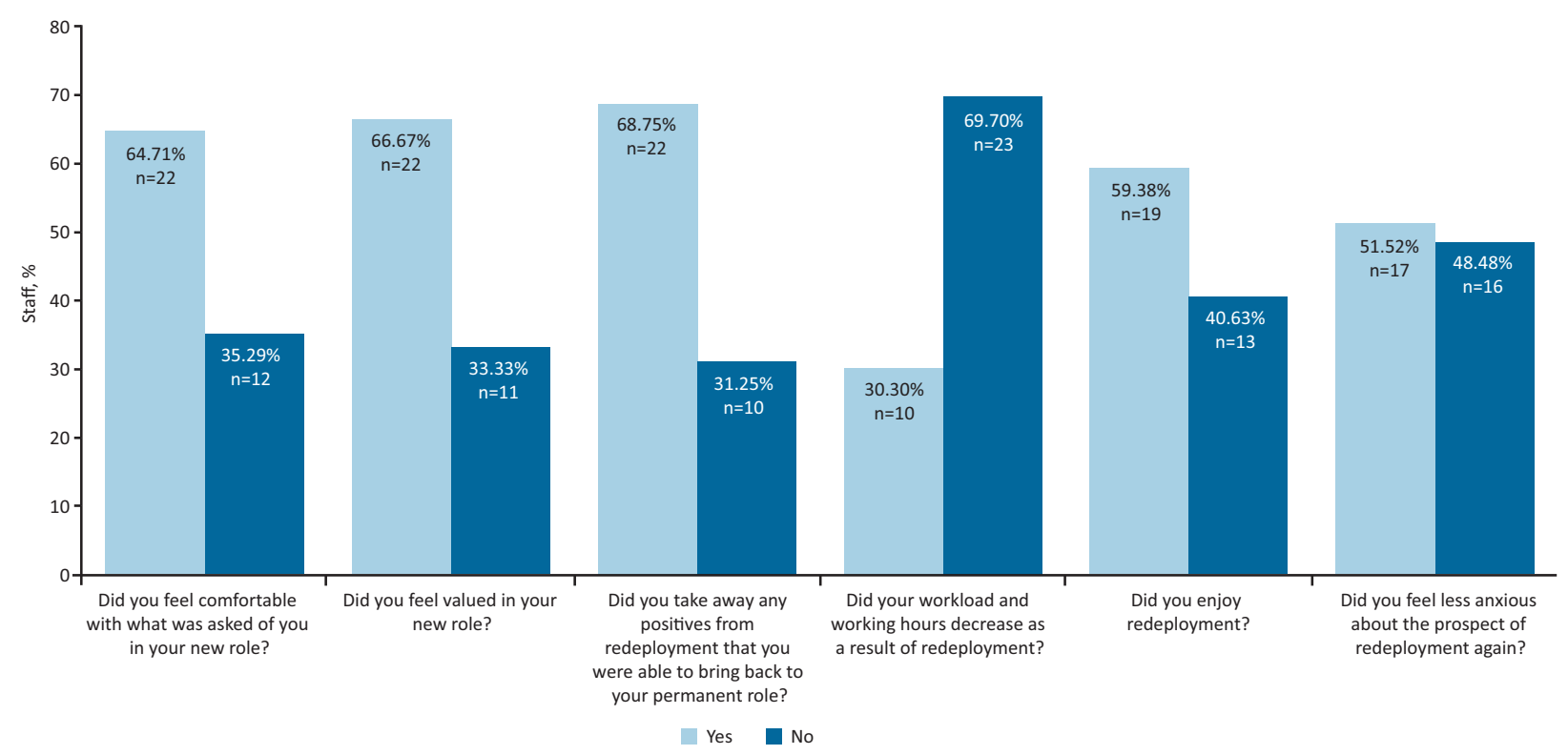

Fig 2. Responses of the redeployed cohort on their experiences of redeployment during the first wave of the COVID-19 pandemic. 


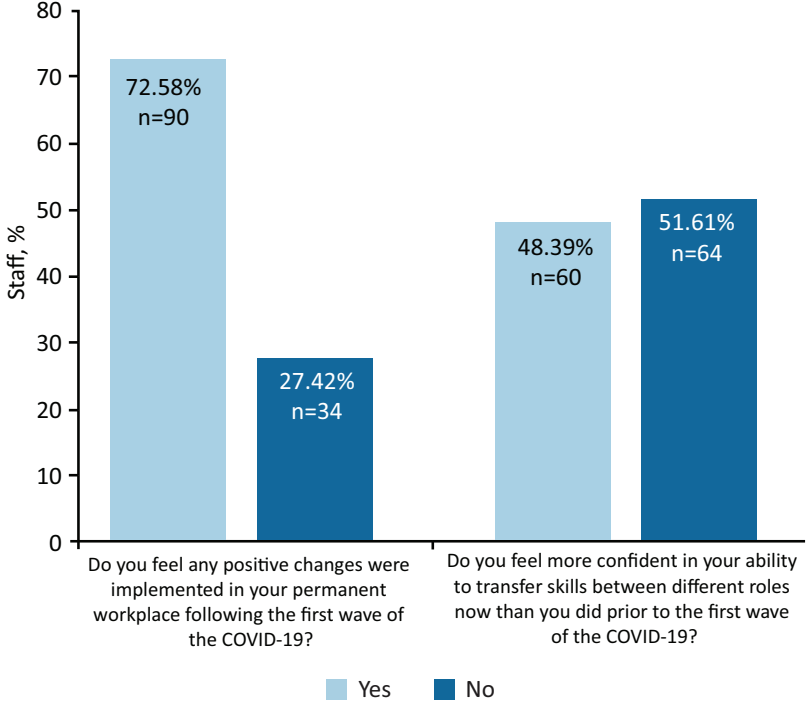

Fig 3. The responses of healthcare professionals regarding positive change in the NHS as a result of the first wave of the COVID-19 pandemic.

permanent role. Significantly, $69.70 \%$ of staff experienced the same or longer working hours as in their prior roles. Importantly, $59.38 \%$ enjoyed their time in redeployment. Notably, however, the results indicate that the experience of redeployment did not lead to significantly lower levels of anxiety with the prospect of a second redeployment $(51.52 \%)$.

The second aim of the study was to establish the perception of both the redeployed and non-redeployed staff on positive change within the NHS following the first wave of the pandemic. This also involved determining whether participants felt more confident in different job roles, as this represents a great advantage in the diverse medical setting.

As many as $72.58 \%$ of participants felt that positive changes were implemented in their permanent workplaces following the first wave of the pandemic, and $48.39 \%$ felt more confident in transferring skills between different job roles (Fig 3).

Finally, the views of all staff on work-life balance, their working environment and the positive change within the NHS were ascertained before the start of the second wave (Fig 4).

A significant majority of participants $(71.43 \%)$ felt that there was more mental health and wellbeing support offered in their workplace than previously, and $53.78 \%$ stated that they now feel more appreciated as an NHS caregiver. Despite the initial supply challenges, $55.46 \%$ felt that the PPE guidance was sufficient to protect against COVID-19 infection regardless of their role. A significant proportion of the staff questioned $(63.56 \%)$ felt a greater sense of fellowship and camaraderie in the workplace compared with a year ago. Over three-quarters (75.63\%) felt that COVID-19 had influenced their perspective on work-life balance and $56.30 \%$ felt that positive change would happen within the NHS as a direct result of the COVID-19 pandemic. Notably, it could be argued that one of the most crucial findings of this survey was that $54.55 \%$ of participants believed that this positive change would be long-lasting.

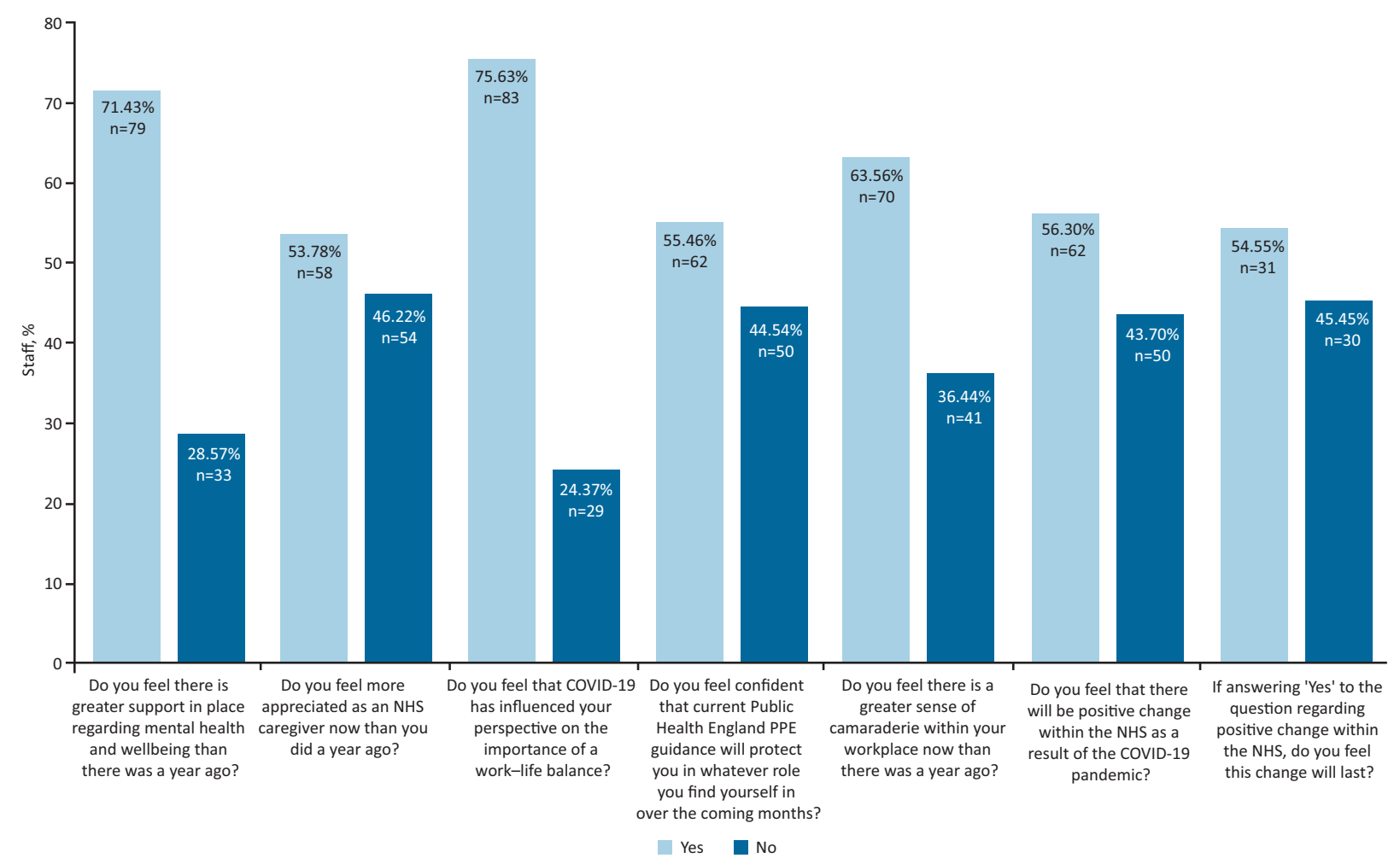

Fig 4. Views of staff regarding workplace change in the context of the second wave of the COVID-19 pandemic. PPE = personal protective equipment. 


\section{Discussion}

Principal findings / comparison to other studies and important differences

Our study probed the experiences of redeployed staff during the first wave of the pandemic and assessed whether any genuine, positive change had occurred. Its goal was to establish whether staff gained transferable skills that would continue to serve them in their permanent roles, and whether their experience of redeployment reduced their anxiety concerning the prospect of further redeployment during a second wave of the COVID-19 pandemic.

The study results indicate that redeployed members of staff felt comfortable and valued in their new roles and that they did have an enjoyable experience of redeployment, despite most working similar or longer hours. These results are concordant with those from a study at McGill University where the majority of redeployed residents were satisfied with their redeployment. ${ }^{16}$ A study on the redeployment of ophthalmologists in the UK demonstrated that they were able to contribute to the care of patients significantly; the ability to take your own niche skills to a new role and implement positive change there may be a notable contributing factor in enjoyment and the feeling of being valued. ${ }^{16}$

Similar to the findings by Payne et al who demonstrated increased confidence in both performing technical and non-technical skills by redeployed surgical trainees, as many as $68.75 \%$ of redeployed members of staff who took part in our survey felt that they had gained positive experiences that will be useful in their permanent roles. ${ }^{17}$ Interestingly, however, when the entire study cohort, including both the non-redeployed and redeployed members of staff, were questioned on whether they felt confident in transferring skills between different roles, the majority answered negatively. This could potentially indicate that the non-redeployed members of staff, who made up the majority of this study, felt less able to transfer between their roles compared with their redeployed counterparts. Moreover, staff did not feel less anxious about the prospect of future redeployment, despite having positive experiences.

When questioned on the introduction of positive change within the NHS, almost $73 \%$ of the participants, including both redeployed and non-redeployed members, answered that they felt positive changes had been implemented. Hence, it would be fair to assume that the aforementioned changes associated with increased workplace support have yielded positive results across the NHS. ${ }^{11-15,18}$ This is furthered by participants reporting feeling more supported and appreciated as NHS caregivers in the face of the second wave of the pandemic, while also enjoying a greater sense of camaraderie in the workplace. The immense value of this cannot be underestimated. Most importantly, staff also believed that further long-lasting positive change would take place within the NHS over the course of the pandemic and be maintained beyond.

\section{Strengths and weaknesses of the study}

This study allowed us to sample a variety of healthcare staff across multiple trusts, who had been both redeployed and remained in their original post. This enabled the study to be carried out efficiently and results were collected anonymously using virtual platforms. Accordingly, the collection of results was not hindered by social-distancing practices.

The main limitation of the study was the relatively small sample size. This limited further statistical analysis from being carried out between subgroups within the study population as it would have resulted in statistically insignificant sample sizes. The optionality of each question in the questionnaire unavoidably introduced differences in the numbers of answers between questions. Hence, the analysis had to be done on a question-to-question basis prohibiting any cross-question analysis where answer trends could be identified. The binomial nature of the question answers limited the ability to assess nuances associated with answers and the extent of the changes reported.

Additionally, in one instance, we noted 34 answers to our question about professionals being comfortable in their new role during the pandemic, despite only 33 people indicating they had been redeployed. On discussion as a group, we intentionally included this data as some medical staff were not redeployed from their department but did have 'new roles'; for example, those in emergency medicine being restructured into 'hot' or 'cold' teams. It is possible that the participant could have deemed their response appropriate and, therefore, their contribution to this question is still a valid response. We could avoid this in the future by using logic checks in our questionnaire that only reveal relevant questions based on the prior answers.

\section{Meaning of the study / unanswered questions and future research}

The results of this study indicate that, even in challenging and unprecedented times such as those of a global pandemic, constructive change can be implemented and positive experiences can be found. Given the knowledge borne from previous pandemics (such as the 2002-2004 SARS outbreak), it could be suggested that healthcare organisations like the NHS were better equipped to provide mental health and wellbeing support to staff. Furthermore, this could have directly influenced the sense of confidence and value that healthcare workers felt during the pandemic. The response from NHS staff since the onset of the pandemic has been praised throughout the country, and the appreciation by the public has been a huge morale booster. The generosity of local businesses with aid (such as meal donations), weekly nation-wide applause and figures such as Captain Sir Thomas Moore have highlighted the gratitude the general public feel towards the NHS.

Future research could include a larger sample size to derive more statistically significant data and expanding the questionnaire could determine more quantitative data regarding the impact of the pandemic on staff mental health. For example, determining how and when staff utilised the mental health services made available to them and what they found most useful.

With a vaccination programme in place and the permanent end of lockdowns potentially in sight, it is crucial that we do not forget that support for mental health and wellbeing was put in place for a reason, and also that we do have a unique ability to transfer skills. We must maintain the sense of camaraderie and fellowship that facilitates us to ensure we always value each other. We must also remember the displays of gratitude that demonstrated and confirmed how appreciated we, as NHS caregivers, truly are.

\section{Supplementary material}

Additional supplementary material may be found in the online version of this article at www.rcpjournals.org/fhj: 
S1 - The survey undertaken by the medical staff at the different healthcare trusts.

\section{References}

1 Statista. Coronavirus (COVID-19) deaths worldwide per one million population as of February 6, 2021, by country. Statista, 2021. www.statista.com/statistics/1104709/coronavirus-deaths-worldwide-per-million-inhabitants [Accessed 6 February 2021].

2 GOV.UK. Coronavirus (COVID-19) in the UK: Healthcare in United Kingdom. GOV.UK, 2021. https://coronavirus.data.gov.uk/details/ healthcare?areaType = overview\&areaName $=$ United $\%$ 20Kingdom [Accessed 6 February 2021].

3 Hall LH, Johnson J, Watt I, Tsipa A, O'Connor DB. Healthcare staff wellbeing, burnout, and patient safety: A systematic review. PLoS One 2016;11:e0159015.

4 West M. What does the 2019 NHS Staff Survey truly tell us about how staff needs are being met? The King's Fund, 2020. www. kingsfund.org.uk/blog/2020/02/2019-nhs-staff-survey-are-staffneeds-being-met

5 Wu P, Fang Y, Guan Z et al. The psychological impact of the SARS epidemic on hospital employees in China: exposure, risk perception, and altruistic acceptance of risk. Can J Psychiatry 2009;54:302-11.

6 Shaukat N, Ali DM, Razzak J. Physical and mental health impacts of COVID-19 on healthcare workers: a scoping review. Int J Emerg Med 2020;13:40

7 Nelson B, Kaminsky DB. COVID-19's crushing mental health toll on health care workers. Cancer Cytopathology 2020;128:597-8.

8 Wong AH, Pacella-LaBarbara ML, Ray JM, Ranney ML, Chang BP. Healing the Healer: Protecting emergency health care workers' mental health during COVID-19. Ann Emerg Med 2020;76:379-84.

9 Liu C-Y, Yang Y-Z, Zhang X-M et al. The prevalence and influencing factors in anxiety in medical workers fighting COVID-19 in China: a cross-sectional survey. Epidemiol Infect 2020;148:e98.
10 Wilson C. Mental health impacts of COVID-19 on NHS healthcare staff. UK Parliament, 2020. https://post.parliament.uk/mentalhealth-impacts-of-covid-19-on-nhs-healthcare-staff [Accessed 6 February 2021].

11 British Medical Association. The mental health and wellbeing of the medical workforce - now and beyond COVID-19. BMA, 2020.

12 NHS England. Support during COVID-19 so far. NHS. www.england. nhs.uk/ournhspeople/online-version/lfaop/support-during-covid [Accessed 6 February 2021].

13 NHS Employers. Health, safety and wellbeing. NHS. www.nhsemployers.org/covid19/health-safety-and-wellbeing/support-availablefor-nhs-staff [Accessed 6 February 2021].

14 Wood S. Caring for our carers: what can COVID-19 teach us about NHS staff wellbeing? The Health Foundation, 2020. www.health. org.uk/news-and-comment/blogs/caring-for-our-carers-whatcan-covid-19-teach-us-about-nhs-staff-wellbeing [Accessed 19 December 2020].

15 Kisely S, Warren N, McMahon L et al. Occurrence, prevention, and management of the psychological effects of emerging virus outbreaks on healthcare workers: rapid review and meta-analysis. BMJ 2020;369:m1642.

16 Lim C, De Silva I, Moussa G et al. Redeployment of ophthalmologists in the United Kingdom during the Coronavirus Disease Pandemic. Eur ] Ophthalmol 2020:1120672120953339.

17 Claudio F, Aalamian A, Cummings B-A et al. Residency redeployment during a pandemic: Lessons for balancing service and learning. Can Med Educ J 2020;11:e132-3.

18 Faderani R, Monks M, Peprah D et al. Improving wellbeing among UK doctors redeployed during the COVID-19 pandemic. FHJ 2020;7:e71-6.

Address for correspondence: Dr Tayla Fay, University College Hospital, 235 Euston Road, London NW1 2BU, UK. Email: taylafay23@gmail.com 